STUPP-96-142

hep-th/9602141

February, 1996

\title{
Ward-Takahashi Identities in Large $N$ Field Theories
}

\author{
Minako Arakif and Yoshiaki Tanil] \\ Physics Department, Faculty of Science \\ Saitama University, Urawa, Saitama 338, Japan
}

\begin{abstract}
The Ward-Takahashi identities in large $N$ field theories are expressed in a simple form using master fields. Operators appearing in these expressions are found to be generators of symmetry transformations acting on the master fields.
\end{abstract}

\footnotetext{
* e-mail: minako@th.phy.saitama-u.ac.jp

$\dagger$ e-mail: tanii@th.phy.saitama-u.ac.jp
} 
The technique of large $N$ limit is useful to study non-perturbative properties of quantum field theories. (For a review and references, see [1] for example.) When dynamical variables are $N \times N$ matrices, only planar Feynman diagrams contribute to the large $N$ limit [2]. Such matrix models in the large $N$ limit were used to study $\mathrm{SU}(N)$ gauge theories and two-dimensional quantum gravity. In studying the large $N$ field theories the idea of master fields was introduced [3]. In the large $N$ limit path integrals are dominated by a single classical configuration called the master field, and all Green's functions are determined by it.

Recently, a mathematically precise formulation of master fields was given [4], [5] using non-commutative variables [6], and was further developed [7]-10]. Similar formulations had previously been discussed in refs. [11], [12]. In this formulation master fields are given in terms of all connected Green's functions. One needs to solve the theories and obtain all Green's functions before constructing the master fields. At present it is not known how to construct master fields explicitly except for a few simple theories such as two-dimensional QCD and zero-dimensional matrix models. It remains to be found a method to construct master fields in more general theories.

On the other hand, one may use master fields to discuss formal properties of the theories. The purpose of this paper is to discuss the Ward-Takahashi identities corresponding to symmetry transformations in the large $N$ field theories. We find that the Ward-Takahashi identities can be expressed in a simple form using master fields. We also find that operators appearing in these expressions are generators of the symmetry transformations acting on the master fields.

Let us first recall the method of master fields in large $N$ field theories [4], [5], [1], [12]. We follow the approach in ref. [12]. We shall consider general $\mathrm{SU}(N)$ invariant field theories in which fields $\Phi_{I}$ take their values in $N \times N$ hermitian matrices. The index $I$ represents a type of the fields as well as space-time coordinates $x^{\mu}$, on which the fields depend. The fields can be either bosonic $\left((-1)^{|I|}=+1\right)$ or fermionic $\left((-1)^{|I|}=-1\right)$. The path integral has a form

$$
\int \mathcal{D} \Phi_{I} \mathrm{e}^{i N S[\Phi]}
$$

where coupling constants in the action $S[\Phi]$ are independent of $N$. We are interested 
in the large $N$ limit of vacuum expectation values

$$
Z_{I_{k} \cdots I_{1}}=\lim _{N \rightarrow \infty} \frac{1}{N}\left\langle 0\left|\operatorname{Ttr}\left(\Phi_{I_{k}} \cdots \Phi_{I_{1}}\right)\right| 0\right\rangle .
$$

In the perturbation theory they are given by a sum of planar Feynman diagrams.

As discussed in ref. [12] it is convenient to introduce a non-commuting source $j_{I}$ for each field $\Phi_{I}$ and consider the generating functional

$$
Z[j]=1+\sum_{k=1}^{\infty} \sum_{\{I\}} j_{I_{1}} \cdots j_{I_{k}} Z_{I_{k} \cdots I_{1}}
$$

If we define the differentiation with respect to the non-commuting sources by

$$
\frac{\delta}{\delta j_{I}}\left(a j_{I_{1}} j_{I_{2}} \cdots j_{I_{k}}\right)=a \delta_{I I_{1}} j_{I_{2}} \cdots j_{I_{k}}, \quad \frac{\delta}{\delta j_{I}} a=0,
$$

where $a$ is a constant, the planar Green's functions (2) are given by

$$
Z_{I_{k} \cdots I_{1}}=\left.\frac{\delta}{\delta j_{I_{k}}} \cdots \frac{\delta}{\delta j_{I_{1}}} Z[j]\right|_{j=0} .
$$

Similarly, the generating functional of connected planar Green's functions is defined by using non-commuting sources $J_{I}$ as

$$
W[J]=\sum_{k=1}^{\infty} \sum_{\{I\}} J_{I_{1}} \cdots J_{I_{k}} \lim _{N \rightarrow \infty} \frac{1}{N}\left\langle 0\left|\mathrm{~T} \operatorname{tr}\left(\Phi_{I_{k}} \cdots \Phi_{I_{1}}\right)\right| 0\right\rangle_{\text {connected }}
$$

In ref. [12] it was shown that these two generating functionals are related by

$$
Z[j]=1+W[J], \quad J_{I}=j_{I} Z[j] .
$$

We define operators $\hat{\Phi}_{I}$ and $\hat{\Pi}_{I}$ by a similarity transformation

$$
\begin{aligned}
& \hat{\Phi}_{I}=Z[j]^{-1} \frac{\delta}{\delta j_{I}} Z[j]=\frac{\delta}{\delta J_{I}}+\frac{\delta W[J]}{\delta J_{I}}, \\
& \hat{\Pi}_{I}=Z[j]^{-1} j_{I} Z[j]=(1+W[J])^{-1} J_{I},
\end{aligned}
$$


which satisfy

$$
\hat{\Phi}_{I} \hat{\Pi}_{J}=\delta_{I J}
$$

In terms of these operators the planar Green's functions (5) can be expressed as

$$
\begin{aligned}
Z_{I_{k} \cdots I_{1}} & =\left.\hat{\Phi}_{I_{k}} \cdots \hat{\Phi}_{I_{1}} \cdot 1\right|_{J=0} \\
& =\left\langle\Omega\left|\hat{\Phi}_{I_{k}} \cdots \hat{\Phi}_{I_{1}}\right| \Omega\right\rangle,
\end{aligned}
$$

where the states $|\Omega\rangle$ and $\langle\Omega|$ are defined by

$$
\frac{\delta}{\delta J_{I}}|\Omega\rangle=0, \quad\langle\Omega| J_{I}=0, \quad\langle\Omega \mid \Omega\rangle=1 .
$$

$\hat{\Phi}_{I}$ are the master field operators discussed in the recent works [4], [5]. The operators $J_{I}$ and $\frac{\delta}{\delta J_{I}}$ correspond to $\hat{a}_{I}^{\dagger}$ and $\hat{a}_{I}$ respectively in the notation of refs. 田, [5]. We note that these operators are not quantum operators but just represent $N \rightarrow \infty$ limit of $N \times N$ matrices.

We now turn to a discussion of the Ward-Takahashi identities. Suppose that there are $n$ conserved charges $Q^{a}(a=1,2, \cdots, n)$ corresponding to symmetry transformations of the theory. These charges generate the transformations of the quantum fields $\Phi_{I}$

$$
\delta \Phi_{I}=\left[i \lambda^{a} Q^{a}, \Phi_{I}\right] \equiv \lambda^{a} R_{I}^{a}(\Phi),
$$

where $\lambda^{a}$ are parameters of the transformations and have the same statistics as $Q^{a}$. They satisfy a commutation relation

$$
\left[Q^{a}, Q^{b}\right\}=i f^{a b c} Q^{c}
$$

where $[A, B\}$ represents an anticommutator of $A$ and $B$ when both of $A$ and $B$ are fermionic, and a commutator otherwise. We assume that the structure constant $f^{a b c}$ is bosonic. From the Jacobi identity

$$
\left[Q^{a},\left[Q^{b}, \Phi_{I}\right\}\right\}-(-1)^{|a||b|}\left[Q^{b},\left[Q^{a}, \Phi_{I}\right\}\right\}=\left[\left[Q^{a}, Q^{b}\right\}, \Phi_{I}\right\},
$$

we obtain a condition on $R_{I}^{a}$

$$
\left[Q^{a}, R_{I}^{b}\right\}-(-1)^{|a||b|}\left[Q^{b}, R_{I}^{a}\right\}=i f^{a b c} R_{I}^{c}
$$


We will use this relation later. When the vacuum is invariant under the symmetry transformations $Q^{a}|0\rangle=0$, we obtain the Ward-Takahashi identities

$$
\begin{aligned}
0 & =\left\langle 0\left|\left[i \lambda^{a} Q^{a}, \operatorname{T} \operatorname{tr}\left(\Phi_{I_{k}} \cdots \Phi_{I_{1}}\right)\right]\right| 0\right\rangle \\
& =\sum_{i=1}^{k}\left\langle 0\left|\operatorname{Tr}\left(\Phi_{I_{k}} \cdots \Phi_{I_{i+1}} \lambda^{a} R_{I_{i}}^{a}(\Phi) \Phi_{I_{i-1}} \cdots \Phi_{I_{1}}\right)\right| 0\right\rangle .
\end{aligned}
$$

These identities are satisfied for an arbitrary $N$.

We would like to express these identities using the master fields $\hat{\Phi}_{I}$ in the large $N$ limit. By taking the limit $N \rightarrow \infty$ and multiplying non-commuting sources we obtain

$$
\sum_{k=1}^{\infty} \sum_{\{I\}} j_{I_{1}} \cdots j_{I_{k}} \sum_{i=1}^{k} \lim _{N \rightarrow \infty} \frac{1}{N}\left\langle 0\left|\mathrm{~T} \operatorname{tr}\left(\Phi_{I_{k}} \cdots \Phi_{I_{i+1}} \lambda^{a} R_{I_{i}}^{a}(\Phi) \Phi_{I_{i-1}} \cdots \Phi_{I_{1}}\right)\right| 0\right\rangle=0
$$

To proceed, we expand the functions $R_{I}^{a}(\Phi)$ as

$$
R_{I}^{a}(\Phi)=R_{I}^{(0) a}+\sum_{K} R_{I, K}^{(1) a} \Phi_{K}+\sum_{K_{1}, K_{2}} R_{I, K_{1} K_{2}}^{(2) a} \Phi_{K_{1}} \Phi_{K_{2}}+\cdots
$$

and consider the $m$-th term. The corresponding term in eq. (17) becomes

$$
\begin{aligned}
& \sum_{k=1}^{\infty} \sum_{i=1}^{k} \sum_{\{I, K\}} j_{I_{1}} \cdots j_{I_{i}} \lambda^{a} R_{I_{i}, K_{1} \cdots K_{m}}^{(m) a} j_{I_{i+1}} \cdots j_{I_{k}} Z_{I_{k}, \cdots, I_{i+1}, K_{m}, \cdots, K_{1}, I_{i-1}, \cdots, I_{1}} \\
& \quad=\sum_{k=1}^{\infty} \sum_{\{I, K\}} j_{I_{1}} \cdots j_{I_{k}} \lambda^{a} R_{I_{k}, K_{1} \cdots K_{m}}^{(m) a} \frac{\delta}{\delta j_{K_{m}}} \cdots \frac{\delta}{\delta j_{K_{1}}} \frac{\delta}{\delta j_{I_{k-1}}} \cdots \frac{\delta}{\delta j_{I_{1}}} Z[j] .
\end{aligned}
$$

Therefore, if we define

$$
\hat{q}^{a}=i \sum_{k=0}^{\infty} \sum_{\{I\}, K}(-1)^{|a|\left(\left|I_{1}\right|+\cdots+\left|I_{k}\right|+|K|\right)} j_{I_{1}} \cdots j_{I_{k}} j_{K} R_{K}^{a}\left(\frac{\delta}{\delta j}\right) \frac{\delta}{\delta j_{I_{k}}} \cdots \frac{\delta}{\delta j_{I_{1}}}
$$

the Ward-Takahashi identities (17) can be rewritten as

$$
\hat{q}^{a} Z[j]=0
$$


By the similarity transformation (8) we further define the master charges

$$
\begin{aligned}
\hat{Q}^{a} & =Z[j]^{-1} \hat{q}^{a} Z[j] \\
& =\sum_{k=0}^{\infty} \sum_{\{I\}}(-1)^{|a|\left(\left|I_{1}\right|+\cdots+\left|I_{k}\right|\right)} \hat{\Pi}_{I_{1}} \cdots \hat{\Pi}_{I_{k}} \hat{S}^{a} \hat{\Phi}_{I_{k}} \cdots \hat{\Phi}_{I_{1}},
\end{aligned}
$$

where

$$
\hat{S}^{a}=i \sum_{K}(-1)^{|a||K|} \hat{\Pi}_{K} R_{K}^{a}(\hat{\Phi})
$$

Then the Ward-Takahashi identities (21) become

$$
\hat{Q}^{a}|\Omega\rangle=0
$$

To understand a physical meaning of the master charges $\hat{Q}^{a}$ appearing in the Ward-Takahashi identities (24) let us compute the algebra among $\hat{Q}^{a}, \hat{\Phi}^{a}$ and $\hat{\Pi}^{a}$. Commutation relations between $\hat{Q}^{a}$ and $\hat{\Phi}^{a}, \hat{\Pi}^{a}$ are easily obtained as

$$
\begin{aligned}
& i\left[\hat{Q}^{a}, \hat{\Phi}_{I}\right\}=-(-1)^{|a||I|} i \hat{\Phi}_{I} \hat{S}^{a}=R_{I}^{a}(\hat{\Phi}), \\
& i\left[\hat{Q}^{a}, \hat{\Pi}_{I}\right\}=i \hat{S}^{a} \hat{\Pi}_{I} .
\end{aligned}
$$

We see that the master charges $\hat{Q}^{a}$ generate the same transformations on the master fields $\hat{\Phi}_{I}$ as the quantum charges $Q^{a}$ generate on the quantum fields $\Phi_{I}$ (12). The commutation relation among $\hat{Q}^{a}$ is found to be

$$
\left[\hat{Q}^{a}, \hat{Q}^{b}\right\}=i f^{a b c} \hat{Q}^{c}
$$

which is the same algebra as that of the quantum charges (13). This can be proved as follows. First we compute

$$
\begin{aligned}
{\left[\hat{Q}^{a}, \hat{Q}^{b}\right\}=} & {\left[\hat{Q}^{a}, \sum_{k=0}^{\infty} \sum_{\{I\}}(-1)^{|b|\left(\left|I_{1}\right|+\cdots+\left|I_{k}\right|\right)} \hat{\Pi}_{I_{1}} \cdots \hat{\Pi}_{I_{k}} \hat{S}^{b} \hat{\Phi}_{I_{k}} \cdots \hat{\Phi}_{I_{1}}\right\} } \\
= & \sum_{k=0}^{\infty} \sum_{\{I\}}(-1)^{(|a|+|b|)\left(\left|I_{1}\right|+\cdots+\left|I_{k}\right|\right)}\left(\hat{\Pi}_{I_{1}} \cdots \hat{\Pi}_{I_{k}}\left[\hat{Q}^{a}, \hat{S}^{b}\right\} \hat{\Phi}_{I_{k}} \cdots \hat{\Phi}_{I_{1}}\right. \\
& +\sum_{i=1}^{k}(-1)^{|a|\left(\left|I_{i}\right|+\cdots+\left|I_{k}\right|\right)} \hat{\Pi}_{I_{1}} \cdots \hat{\Pi}_{I_{i-1}} \hat{S}^{a} \hat{\Pi}_{I_{i}} \cdots \hat{\Pi}_{I_{k}} \hat{S}^{b} \hat{\Phi}_{I_{k}} \cdots \hat{\Phi}_{I_{1}} \\
& \left.-\sum_{i=1}^{k}(-1)^{|a|\left(|b|+\left|I_{i}\right|+\cdots+\left|I_{k}\right|\right)} \hat{\Pi}_{I_{1}} \cdots \hat{\Pi}_{I_{k}} \hat{S}^{b} \hat{\Phi}_{I_{k}} \cdots \hat{\Phi}_{I_{i}} \hat{S}^{a} \hat{\Phi}_{I_{i-1}} \cdots \hat{\Phi}_{I_{1}}\right),
\end{aligned}
$$


where $k=0$ terms for the second and the third terms in the last equality are defined to be zero. We have used the commutation relations (25). By rearranging the summations and using the definition of $\hat{Q}^{a}$ in eq. (22), we obtain

$$
\begin{aligned}
{\left[\hat{Q}^{a}, \hat{Q}^{b}\right\}=} & \sum_{k=0}^{\infty} \sum_{\{I\}}(-1)^{(|a|+|b|)\left(\left|I_{1}\right|+\cdots+\left|I_{k}\right|\right)} \hat{\Pi}_{I_{1}} \cdots \hat{\Pi}_{I_{k}} \\
& \times\left(\left[\hat{Q}^{a}, \hat{S}^{b}\right\}-(-1)^{|a||b|}\left[\hat{Q}^{b}, \hat{S}^{a}\right\}-\left[\hat{S}^{a}, \hat{S}^{b}\right\}\right) \hat{\Phi}_{I_{k}} \cdots \hat{\Phi}_{I_{1}} .
\end{aligned}
$$

Substituting eq. (23) and using eqs. (25), (15), the expression in the brackets in eq. (28) becomes

$$
\begin{aligned}
i \sum_{K} & (-1)^{|K|(|a|+|b|)} \hat{\Pi}_{K}\left(\left[\hat{Q}^{a}, R_{K}^{b}(\hat{\Phi})\right\}-(-1)^{|a||b|}\left[\hat{Q}^{b}, R_{K}^{a}(\hat{\Phi})\right\}\right) \\
\quad & =-f^{a b c} \sum_{K}(-1)^{|K||c|} \hat{\Pi}_{K} R_{K}^{c}(\hat{\Phi}) \\
& =i f^{a b c} \hat{S}^{c} .
\end{aligned}
$$

Substituting this result back in eq. (28) we obtain the commutation relation (26).

To summarize, we have shown that the Ward-Takahashi identities in large $N$ field theories can be expressed as eq. (24) using the master fields. The master charges $\hat{Q}^{a}$ satisfy the commutation relations (25) and (26), and are generators of the symmetry transformations realized on the space of master fields. We should note that the existence of symmetry generators for master fields is not an obvious fact. It is somewhat surprising that the same algebra can be realized on the space of master fields as on the space of quantum operators because master field operators just represent $N \rightarrow \infty$ limit of $N \times N$ matrices and have nothing to do with quantum operators.

Our formula of the master charges (22) gives, as a special case, the spacetime translation generator (momentum operator) $\hat{P}^{\mu}$ for master fields, which was given in eq. (5.6) of ref. [5]. In this case the operator in eq. (20) commutes with $Z(j)$, and therefore coincides with the master charge in eq. (22).

In the case of $\mathrm{SU}(N)$ gauge theories the most fundamental symmetry is the BRST symmetry. According to the above general result we can construct the master charge $\hat{Q}_{\mathrm{B}}$ for the BRST symmetry, which generates the BRST transformation of master fields. The BRST transformation of master fields was previously discussed in ref. [10] in another context. 


\section{References}

[1] S. Coleman, in Pointlike Structures Inside and Outside Hadrons, ed. A. Zichichi (Plenum Press, 1982, New York and London); The Large N Expansion in Quantum Field Theory and Statistical Physics, eds. E. Brézin and S.R. Wadia, (World Scientific, 1993, Singapore).

[2] G. 't Hooft, Nucl. Phys. B72 (1974) 461.

[3] E. Witten, in Recent Developments in Gauge Theories, eds. G. 't Hooft et. al. (Plenum Press, 1980, New York and London)

[4] M.R. Douglas, Nucl. Phys. B (Proc. Suppl.) 41 (1995) 66; Phys. Lett. B344 (1995) 117.

[5] R. Gopakumar and D.J. Gross, Nucl. Phys. B451 (1995) 379.

[6] D.V. Voiculescu, K.J. Dykema and A. Nica, Free Random Variables, (American Mathematical Society, 1992, Providence).

[7] M.R. Douglas and M. Li, Phys. Lett. B348 (1995) 360.

[8] D. Minic, preprints CCNY-HEP 1/95, hep-th/9502117; CCNY-HEP 3/95, hep-th/9503203.

[9] L. Accardi, I. Aref'eva and I. Volovich, preprint hep-th/9402092; L. Accardi, Y.G. Lu and I. Volovich, preprint hep-th/9412246; L. Accardi, I. Aref'eva, S. Kozirev and I. Volovich, Mod. Phys. Lett. A10 (1995) 2323.

[10] I. Aref'eva and I.V. Volovich, preprint SMI-25-95, hep-th/9510210.

[11] O. Haan, Z. Physik C6 (1980) 345.

[12] P. Cvitanović, Phys. Lett. B99 (1981) 49; P. Cvitanović, P.G. Lauwers and P.N. Scharbach, Nucl. Phys. B203 (1982) 385. 